\title{
The application of function P-sets in band information law hiding
}

$$
\text { Chengxian Fan }{ }^{1,} \text {, Faliang Chang }{ }^{2, b}
$$

${ }^{1}$ School of Electrical Engineering, Shandong University, Jinan, Shandong, 250061 China

${ }^{2}$ School of Control Science and Engineering, Shandong University, Jinan, Shandong, 250061 China

aemail: fcxmar@sdu.edu.cn, bemail:flchang@sdu.edu.cn

Keywords: band information law; information law hiding; recovery theorem

\begin{abstract}
Using the structure, law and dynamic characteristic of function P-sets, the concepts of P-information law and band information law and their generation are given. Then the attribute theorem of P-information, the hiding and hiding theorem of P-information in band information law and the recovery theorem of information law are presented. Last using the above theory results, an application example of information law hiding is given and identified by experiment.
\end{abstract}

\section{Introduction}

Using the discrete values $x_{1}, x_{2}, \cdots, x_{n}$, the function $w(x)=a_{n-1} x^{n-1}+a_{n-2} x^{n-2}+\cdots+a_{1} x+a_{0}$ is generated by interpolation method. Here there are two questions to need to be solved: (1) what kind of mathematical method is used to hide information law $w(x)$ to prevent from being stolen and distorted by others? (2) what kind of mathematics method is used to restore information law $w(x)$ hidden? In the paper, a novel mathematical theory will be used to give the answers about the questions, which is function P-sets ${ }^{[1,2]}$ and has been applied in many research areas ${ }^{[3-6]}$. Function P-sets is the function form of P-sets ${ }^{[7-9]}$.

The generation of band information law $<a, w(x)^{\bar{F}}, b, w(x)^{F}>$

Given function set $S=\left\{s_{1}, s_{2}, \cdots, s_{q}\right\}$, and $\alpha=\left\{\alpha_{1}, \quad \alpha_{2}, \cdots, \alpha_{k}\right\}$ is the attribute set of $S . y_{i}$ is the discrete distribution data set of $\forall s_{i} \in S, \quad i=1,2, \cdots, q$, and

$$
y_{i}=\left\{y_{i, 1}, y_{i, 2}, \cdots, y_{i, n}\right\}
$$

Using $y_{i}$ to get the discrete data set $y$ of $S$, and

$$
y=\left\{y_{1}, y_{2}, \cdots, y_{n}\right\}=\left\{\sum_{i=1}^{q} y_{i, 1}, \sum_{i=1}^{q} y_{i, 2}, \cdots, \sum_{i=1}^{q} y_{i, n}\right\}
$$

Using the data points $\left(x_{1}, y_{1}\right),\left(x_{2}, y_{2}\right), \cdots,\left(x_{n}, y_{n}\right)$ and Lagrange interpolation function $w(x)=$ $\sum_{j=1}^{n} y_{j} \prod_{\substack{i, j=1 \\ i \neq j}}^{n} \frac{x-x_{i}}{x_{j}-x_{i}}$ to get $w(x)=a_{n-1} x^{n-1}+a_{n-2} x^{n-2}+\cdots+a_{1} x+a_{0}$, and $w(x)$ is called the information law generated by $y$, which is shown with dotted line in Fig.1.

$\alpha_{i}$ is supplemented into $\alpha$, which constitute attribute set $\Delta \alpha$, then $\alpha$ becomes $\alpha^{F}$, and

$$
\alpha^{F}=\alpha \cup \Delta \alpha
$$

After supplementing some attributes into $\alpha, S$ becomes function internal P-set $S^{\bar{F}}$, and

$$
S^{\bar{F}}=\left\{s_{1}, s_{2}, \cdots, s_{p}\right\}
$$

Using the similar method to equations (1) and (2) to get the discrete data set $y^{\bar{F}}$ of $S^{\bar{F}}$, and

$$
y^{\bar{F}}=\left\{y_{1}{ }^{\overline{ }}, y_{2}{ }^{\bar{f}}, \cdots, y_{n}{ }^{\bar{f}}\right\}=\left\{\sum_{i=1}^{p} y_{i, 1}, \sum_{i=1}^{p} y_{i, 2}, \cdots, \sum_{i=1}^{p} y_{i, n}\right\}
$$

Using the data points $\left(x_{1}, y_{1}^{\bar{T}}\right),\left(x_{2}, y_{2}^{\bar{T}}\right), \cdots,\left(x_{n}, y_{n}^{\bar{T}}\right)$ and Lagrange interpolation function to get $w(x)^{\bar{F}}=b_{n-1} x^{n-1}+b_{n-2} x^{n-2}+\cdots+b_{1} x+b_{0}$ and is called internal P-information law as shown in Fig.1. 
$\alpha_{j}$ is deleted from $\alpha$, which constitutes attribute set $\nabla \alpha$, then $\alpha$ becomes $\alpha^{\bar{F}}$

$$
\alpha^{\bar{F}}=\alpha-\nabla \alpha
$$

After deleting some attributes from $\alpha$, general function set $S$ becomes function outer P-set $S^{F}$,

$$
S^{F}=\left\{s_{1}, s_{2}, \cdots, s_{r}\right\}
$$

Using the similar method to equations (1) and (2) to get the discrete data set $y^{F}$ of $S^{F}$, and

$$
y^{F}=\left\{y_{1}{ }^{f}, y_{2}{ }^{f}, \cdots, y_{n}{ }^{f}\right\}=\left\{\sum_{i=1}^{r} y_{i, 1}, \sum_{i=1}^{r} y_{i, 2}, \cdots, \sum_{i=1}^{r} y_{i, n}\right\}
$$

Using the data points $\left(x_{1}, y_{1}^{f}\right),\left(x_{2}, y_{2}^{f}\right), \cdots,\left(x_{n}, y_{n}^{f}\right)$ and Lagrange interpolation function to get $w(x)^{F}=c_{n-1} x^{n-1}+c_{n-2} x^{n-2}+\cdots+c_{1} x+c_{0}$ and is called outer P-information law as shown in Fig.1.

Definition 1 The information law pair consisted of $w(x)^{\bar{F}}$ and $w(x)^{F}$ is called P-information law generated by function P-sets $\left(S^{\bar{F}}, S^{F}\right)$, and

$$
\left(w(x)^{\bar{F}}, w(x)^{F}\right)
$$

Definition $2<a, w(x)^{\bar{F}}, b, w(x)^{F}>$ is called band information law generated by $\left(w(x)^{\bar{F}}, w(x)^{F}\right)$, if $w(x)^{\bar{F}}$ and $w(x)^{F}$ are the up-boundary and down-boundary, respectively. $a$ and $b$ are two different common points on x-axis of $<a, w(x)^{\bar{F}}, b, w(x)^{F}>$, respectively.

Theorem 1 If $w(x)_{\lambda}^{\bar{F}}$ is the internal P-information law generated by function internal P-set $S_{\lambda}^{\bar{F}}$, then there exists attribute set $\Delta \alpha \neq \varnothing$ to make $\Delta \alpha$, attribute set $\alpha_{\lambda}^{F}$ of $w(x)_{\lambda}^{\bar{F}}$ and attribute set $\alpha$ of $w(x)$ satisfy the following equation.

$$
\left(\alpha_{\lambda}^{F}-\Delta \alpha\right)-\alpha=\varnothing
$$

Theorem 2 If $w(x)_{\lambda}^{F}$ is the outer P-information law generated by function outer P-set $S_{\lambda}^{F}$, then there exists attribute set $\nabla \alpha \neq \varnothing$ to make $\nabla \alpha$, attribute set $\alpha_{\lambda}^{\bar{F}}$ of $w(x)_{\lambda}^{F}$ and attribute set $\alpha$ of $w(x)$ satisfy the following equation.

$$
\left(\alpha_{\lambda}^{\bar{F}} \cup \nabla \alpha\right)-\alpha=\varnothing
$$

Theorem 3 If $\left\langle a, w(x)_{\lambda}^{\bar{F}}, b, w(x)_{\lambda}^{F}\right\rangle$ is the band information law generated by $\left(w(x)_{\lambda}^{\bar{F}}, w(x)_{\lambda}^{F}\right)$, the sufficient and necessary condition of information law hidden in $\left\langle a, w(x)_{\lambda}^{\bar{F}}, b, w(x)_{\lambda}^{F}\right\rangle$ is that attribute set $\alpha_{\lambda}^{F}$ and $\alpha, \alpha_{\lambda}^{\bar{F}}$ and $\alpha$ satisfy equations (12) and (13), respectively.

$$
\begin{aligned}
& \alpha_{\lambda}^{F}=\alpha \bigcup \Delta \alpha \\
& \alpha_{\lambda}^{\bar{F}}=\alpha-\nabla \alpha
\end{aligned}
$$

Theorem 4 The sufficient and necessary condition of $\left\langle a, w(x)_{\lambda}^{\bar{F}}, b, w(x)_{\lambda}^{F}\right\rangle$ being restored to $w(x)$ is that the attribute set $\alpha, \alpha_{\lambda}^{F}$ and $\alpha_{\lambda}^{\bar{F}}$ satisfy equations (14) and (15), respectively.

$$
\begin{aligned}
& \alpha=\alpha_{\lambda}^{F}-\Delta \alpha \\
& \alpha=\alpha_{\lambda}^{\bar{F}} \cup \nabla \alpha
\end{aligned}
$$

\section{The application of information law hiding}

The hiding-reduction of $w(x)$ in $\left\langle a, w(x)^{\bar{F}}, b, \quad w(x)^{F}>\right.$ and its application.

Given discrete data set $y$, which are the discrete values at $t=1,2,3,4,5,6$ about important information law $w(x)$, and $\alpha$ is the attribute set of $w(x)$. The name of $\alpha_{i} \in \alpha$ is omitted.

$$
\begin{aligned}
y=\left\{y_{1}, y_{2}, y_{3}, y_{4}, y_{5}, y_{6}\right\} & =\{1.1000,1.2600,1.6300,1.4000,1.3100,1.5800\} \\
\alpha & =\left\{\alpha_{1}, \alpha_{2}, \alpha_{3}, \alpha_{4}\right\}
\end{aligned}
$$

Given $\eta_{i} \in(0,1), \quad i=1,2,3,4$, and $\sum_{i=1}^{4} \eta_{i}=1 . \quad \eta_{i}$ is the separation coefficient, and $\eta_{1}=0.18$, $\eta_{2}=0.27, \eta_{3}=0.35, \eta_{4}=0.20$. Using $\eta_{1} \sim \eta_{4}$ to separate $y_{i} \in y$ into $y_{i, 1}=\eta_{1} y_{i}, y_{i, 2}=\eta_{2} y_{i}, \quad y_{i, 3}=$ $\eta_{3} y_{i}, \quad y_{i, 4}=\eta_{4} y_{i}, \quad i=1,2,3,4,5,6$. The separation of $y_{i}$ about $\eta_{i}$ is listed in the Table 1. 
Table 1. The separation of discrete value $y_{i}$ about $\eta_{i}$ of information law $w(x)$ with attribute set

\begin{tabular}{ccccccc}
\hline$k$ & 1 & 2 & 3 & 4 & 5 & 6 \\
\hline$y_{i, 1}$ & 0.198 & 0.227 & 0.293 & 0.252 & 0.236 & 0.284 \\
$y_{i, 2}$ & 0.297 & 0.340 & 0.440 & 0.378 & 0.354 & 0.427 \\
$y_{i, 3}$ & 0.385 & 0.441 & 0.571 & 0.490 & 0.459 & 0.553 \\
$y_{i, 4}$ & 0.220 & 0.252 & 0.326 & 0.280 & 0.262 & 0.316 \\
\hline
\end{tabular}

The data in the Table 1 and $y_{i}$ in equation (9) satisfy $y_{i}=y_{i, 1}+y_{i, 3}+y_{i, 4}+y_{i, 4}=\sum_{j=1}^{4} y_{i, j}$.

$\alpha_{5}, \alpha_{6}, \alpha_{7}$ are supplemented into $\alpha$, or $\alpha_{1}, \alpha_{3}$ are deleted from $\alpha$, then $\alpha$ becomes $\alpha_{i}^{F}, \alpha_{j}^{\bar{F}}$, respectively.

$$
\begin{aligned}
\alpha_{i}^{F}=\alpha \bigcup\left\{\alpha_{5}, \alpha_{6}, \alpha_{7}\right\} & =\left\{\alpha_{1}, \alpha_{2}, \alpha_{3}, \alpha_{4}, \alpha_{5}, \alpha_{6}, \alpha_{7}\right\} \\
\alpha_{j}^{F} & =\alpha-\left\{\alpha_{1}, \alpha_{3}\right\}=\left\{\alpha_{2}, \alpha_{4}\right\}
\end{aligned}
$$

$y_{i, 3}, y_{i, 4}$ are deleted from Table 1 , then $y$ becomes $y_{i}^{\bar{F}}$, and Table 2 is obtained.

Table 2. The separation of discrete value $y_{i}$ about $\eta_{i}$ of $w(x)_{i}^{\bar{F}}$ with attribute set $\alpha_{i}^{F}$

\begin{tabular}{ccccccc}
\hline$k$ & 1 & 2 & 3 & 4 & 5 & 6 \\
\hline$y_{i, 1}$ & 0.198 & 0.227 & 0.293 & 0.252 & 0.236 & 0.284 \\
$y_{i, 2}$ & 0.297 & 0.340 & 0.440 & 0.378 & 0.354 & 0.427 \\
\hline
\end{tabular}

From Table 2 and equation (5), $y_{i}^{\overline{\bar{F}}}$ is obtained, and

$$
\begin{aligned}
y_{i}^{\bar{F}} & =\left\{y_{1}{ }^{\overline{ }}, y_{2}{ }^{\bar{f}}, y_{3}{ }^{\overline{ }}, y_{4}{ }^{\bar{f}}, y_{5}{ }^{\overline{ }}, y_{6}{ }^{\bar{f}}\right\} \\
& \left.=\left(y_{1,1}+y_{1,2}\right),\left(y_{2,1}+y_{2,2}\right),\left(y_{3,1}+y_{3,2}\right),\left(y_{4,1}+y_{4,2}\right),\left(y_{5,1}+y_{5,2}\right),\left(y_{6,1}+y_{6,2}\right)\right\} \\
& =\{0.4950,0.5670,0.7335,0.6300,0.5895,0.7110\}
\end{aligned}
$$

$y_{i, 5}, y_{i, 6}, y_{i, 7}$ are supplemented into table 1 , then $y$ becomes $y_{j}^{F}$, and Table 3 is obtained.

Table 3. The separation of discrete value $y_{i}$ about $\eta_{i}$ of $w(x)_{j}^{F}$ with attribute set $\alpha_{j}^{\bar{F}}$

\begin{tabular}{ccccccc}
\hline$k$ & 1 & 2 & 3 & 4 & 5 & 6 \\
\hline$y_{i, 1}$ & 0.198 & 0.227 & 0.293 & 0.252 & 0.236 & 0.284 \\
$y_{i, 2}$ & 0.297 & 0.340 & 0.440 & 0.378 & 0.354 & 0.427 \\
$y_{i, 3}$ & 0.385 & 0.441 & 0.571 & 0.490 & 0.459 & 0.553 \\
$y_{i, 4}$ & 0.220 & 0.252 & 0.326 & 0.280 & 0.262 & 0.316 \\
$y_{i, 5}$ & 0.312 & 0.121 & 0.271 & 0.168 & 0.140 & 0.230 \\
$y_{i, 6}$ & 0.127 & 0.239 & 0.193 & 0.270 & 0.283 & 0.379 \\
$y_{i, 7}$ & 0.200 & 0.093 & 0.237 & 0.128 & 0.377 & 0.071 \\
\hline
\end{tabular}

From Table 3 and equation (8), $y_{j}^{F}$ is obtained, and

$$
\begin{aligned}
y_{j}^{F} & =\left\{y_{1}{ }^{f}, y_{2}{ }^{f}, y_{3}{ }^{f},{y_{4}}^{f}, y_{5}{ }^{f}, y_{6}{ }^{f}\right\} \\
& =\left\{\left(y_{1,1}+y_{2,1}+y_{3,1}+y_{4,1}+y_{5,1}+y_{6,1}+y_{7,1}\right), \cdots,\left(y_{1,6}+y_{2,6}+y_{3,6}+y_{4,6}+y_{5,6}+y_{6,6}+y_{7,6}\right)\right. \\
& =\{1.7392,1.7119,2.3304,1.9658,2.1093,2.2598\}
\end{aligned}
$$

Obviously, $w(x), \quad w(x)_{i}^{\bar{F}}$ and $w(x)_{j}^{F}$ generated by $y_{j}^{F}$ satisfy $w(x)_{i}^{\bar{F}}<w(x)<w(x)_{j}^{F}$, or $w(x)$ is hidden in band information law $<a, w(x)_{i}^{\bar{F}}, b, w(x)_{j}^{F}>$, which is shown in Fig.1. 


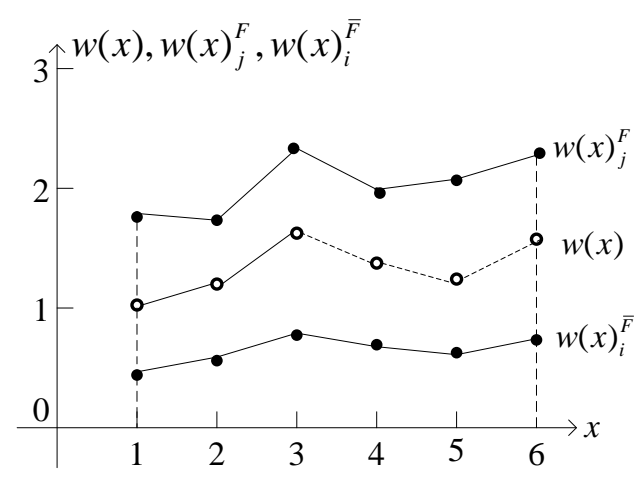

Fig.1 $w(x)_{i}^{\bar{F}}$ and $w(x)_{j}^{F}$ are expressed by real line. Information law $w(x)$ is hidden in $\left\langle a, w(x)_{i}^{\bar{F}}, b, w(x)_{i}^{F}\right\rangle$, and expressed by dotted lin, and $a=1, b=6$.

\section{The experimental verification of information law $w(x)$ reduction.}

Data set $y$ is obtained by the discretization to $w(x)$. Using the structure and characteristic of function P-sets: if attribute set $\alpha$ is supplemented into some attributes, the elements in $y$ will be reduced. If some attributes are deleted from $\alpha$, the elements in $y$ will be added, so $<a, w(x)_{i}^{\bar{F}}, b, w(x)_{j}^{F}>$ is obtained. Using theorem $4,\left\langle a, w(x)_{i}^{\bar{F}}, b, w(x)_{j}^{F}>\right.$ is restored to $w(x)$. The error between $w(x)$ obtained by reduction and $w(x)$ generated by equation (16) is $0.0127 \%$.

\section{Conclusion}

Information law $w(x)$ is hidden in band information law $<a, w(x)^{\bar{F}}, b, w(x)^{F}>$, and $<a, w(x)^{\bar{F}}, b$, $w(x)^{F}>$ is not single, so it is difficult to steal $w(x)$ from $\left\langle a, w(x)^{\bar{F}}, b, w(x)^{F}>\right.$. This is because people do not known which attributes are supplemented into, and which attributes are deleted from $\alpha$, even the definition form and structure of $\alpha_{i}$. It is of no avail to steal $w(x)$ from $<a, w(x)^{\bar{F}}, b, w(x)^{F}>$ by classical mathematical methods, which is proved by many experiments. The study given in the paper is a novel application of function P-sets in dynamic information system.

\section{References}

[1]Shi Kaiquan. Function P-sets[J]. International Journal of Machine Learning and Cybernetic, 2011, 2(4): 281-288.

[2]Shi Kaiquan. Function P-sets[J]. Journal of Shandong University(Natural Science), 2011, 46(2): 62-69 (in Chinese).

[3] Shi Kaiquan. P-information law intelligent fusion and soft information image intelligent generation, Journal of Shandong University(Natural Science), 2014, 49(4): 1-17(in Chinese).

[4]Lin Rong, Fan Chengxian. Function P-sets and dynamic characteristics of information regularity[J]. Journal of Shandong University(Natural Science), 2012, 47(1), 121-126(in Chinese).

[5]Fan Chengxian, Liu Shuqin Li Kejun. P-reasoning and the status information identification of power system. Energy Education Science and Technology Part A: Energy Science and Research 2013, 30(2), 1023-1030.

[6]Zhao Wenju, Fan Chengxian. Attribute dependence of function P-sets and its application. Journal of Shandong University (Natural Science), 2011,46(6): 115-120(in Chinese).

[7]Shi Kaiquan. P-sets and its applications. International Journal Advances in Systems Science and Applications, 2009, 9(2): 209-219.

[8]Fan Chengxian, Lin Hongkang. P-sets and the reasoning- identification of disaster information. 
An International Journal of Convergence Information Technology, 2012, 7(1):337-345.

[9] Shi Kaiquan. P-sets, inverse P-sets and the intelligent fusion-filter identification of information. Computer Science, 2012, 39(4): 1-13 (in Chinese). 